RICYDE. Revista Internacional de Ciencias del Deporte doi: $10.5232 /$ ricyde

Rev. int. cienc. deporte

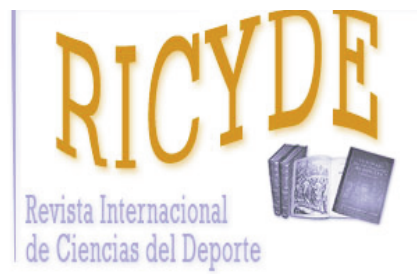

RICYDE. Revista Internacional de Ciencias del Deporte VOLUMEN XIV - AÑO XIV

Páginas:233-242 ISSN:1885-3137

Número: 53 - Julio - 2018

\title{
Explorando el efecto del contacto y el deporte inclusivo en Educación Física en las actitudes hacia la discapacidad intelectual en estudiantes de secundaria \\ Exploring the effect of contact and inclusive sport on Physical Education in the attitudes toward intellectual disability of high school students
}

\author{
Jorge Abellán1, Nieves Sáez-Gallego1, Raúl Reina²
}

1.Universidad Castilla-La Mancha. España

2.Universidad Miguel Hernández de Elche. España

\section{Resumen}

Los programas de cambio de actitudes hacia la discapacidad han venido utilizando, entre otros, la práctica de deporte inclusivo y el contacto con personas del colectivo de referencia. A pesar de su presencia en la sociedad, apenas existen trabajos en los que se emplee el contacto con personas con discapacidad intelectual como medio para cambiar las actitudes hacia la discapacidad. Este trabajo examina el efecto de un programa que utiliza el deporte inclusivo y el contacto con personas con discapacidad intelectual en las actitudes hacia la discapacidad de estudiantes de Secundaria durante las clases de Educación Física. Ochenta y tres estudiantes de ESO de dos centros de Castilla - La Mancha (15,18 \pm 0,89 años) formaron parte de este estudio. Las actitudes de los alumnos, antes y después de la intervención, se registraron mediante los cuestionarios "The Attitudes towards Inclusion of Students with Disabilities in Physical Education" (AISDPE) y la "Escala de Actitud hacia el Alumnado con Discapacidad en Educación Física" (EAADEF). Los resultados muestran que el grupo que realizó deporte inclusivo y una visita a las instalaciones de trabajo de las personas con discapacidad intelectual mejoró en la dimensión cog-

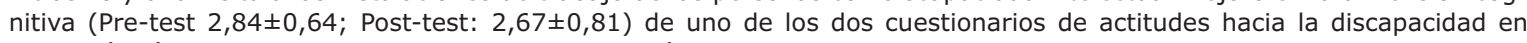
Educación Física utilizados. Parece que un contacto más cercano, compartiendo experiencias y deporte con personas con discapacidad puede mejorar las actitudes hacia la discapacidad en estudiantes de Educación Secundaria.

Palabras clave: inclusión; actitudes, discapacidad intelectual; educación física.

\section{Abstract}

Programs to change attitudes towards disability have been used the practice of inclusive sport and contact with people with disabilities. Despite their presence in society, there are hardly any jobs in which contact with people with intellectual disabilities is used as a means to change attitudes toward disability. The aim of this study is to examine the effect of a Physical Education program using inclusive sport and contact with people with intellectual disabilities on the attitudes of secondary school students towards disability. Eighty-three $(\mathrm{N}=83)$ Compulsory Secondary Education students from two centers in Castilla - La Mancha (15.18 \pm 0.89 years) were part of this study. The students' attitudes, before and after the intervention, were captured through the questionnaires "The Attitudes towards Inclusion of Students with Disabilities in Physical Education" (AISDPE) and the "Attitude Scale for Students with Disability in Physical Education" (EAADEF). The results show that the group that carried out an inclusive sport and a visit to the workplaces of people with intellectual disabilities improves the cognitive dimension (Pre-test 2.84 \pm 0.64 ; Post-test: $2.67 \pm 0.81$ ) in one of the two questionnaires of attitudes toward Physical Education disability used. It seems that an intimate contact, sharing experiences and sports with people with disabilities may improve the attitudes towards the disability of the secondary school students.

Key words: inclusion; attitudes; intellectual disability; physical education. 
Abellán, J.; Sáez-Gallego, N., y Reina, R. (2018). Explorando el efecto del contacto y el deporte inclusivo en Educación Física en las actitudes hacia la discapacidad intelectual de los estudiantes de secundaria. RICYDE. Revista internacional de ciencias del deporte. 53(14), 233-242. https://doi.org/10.5232/ricyde2018.05304

\section{Introducción}

$\mathrm{P}$ rogramas de cambio de actitudes hacia la discapacidad en Educación Física (EF) han demostrado su eficacia en un conjunto de trabajos realizados en los últimos años (Felipe y Garoz, 2014), no reportándose investigaciones previas con resultados negativos en la modificación de las actitudes hacia la discapacidad por medio de la actividad física o la EF. Fundamentalmente han sido dos los tipos de programas utilizados para tratar de modificar las actitudes hacia las personas con discapacidad en EF: programas del día paralímpico en la escuela (Paralympic School Day; IPC, 2004) y el efecto de unidades didácticas de deportes adaptados o juegos sensibilizadores. Por ejemplo McKay, Block y Park (2015) mostraron que una sola jornada de práctica de deportes paralímpicos suponía que los alumnos participantes (de sexto curso de Educación Primaria) mejoraban sus actitudes hacia la inclusión de alumnos con discapacidad en EF. Por otro lado, González y Baños (2012) y Santana y Garoz (2013) utilizaron unidades didácticas de deportes adaptados de boccia (González y Baños, 2012) y fútbol sala, boccia, goalball, atletismo y baloncesto en silla de ruedas (Santana y Garoz, 2013) que modificaron positivamente las actitudes hacia la discapacidad de los estudiantes de Secundaria participantes. Además, Robles-Rodríguez, Abad-Robles, Giménez y BenitoPeinado (2017) mostraron que la opinión de estudiantes de Bachillerato respecto a los paradeportes, entendidos como deportes específicos para una población con discapacidad, era que fomentaban más la igualdad de oportunidades, el respeto y la sensibilización social que deportes tradicionales o no adaptados. Respecto a los juegos motores sensibilizadores, Abellán y Hernández-Martínez (2016) mostraron que, tras su práctica, los maestros en formación presentaban una tendencia a mejorar sus actitudes hacia la discapacidad.

La teoría del contacto (Allport, 1954) indica que se pueden superar los prejuicios hacia un grupo con riesgo de exclusión social a partir del contacto directo. Esta teoría se ha utilizado como telón de fondo para diseñar programas de intervención que mejoren las actitudes hacia la discapacidad. Por ejemplo, Ocete, Pérez-Tejero y Coterón (2015) presentaron una propuesta de intervención para mejorar las posibilidades de inclusión en alumnos de Secundaria en EF a partir de la utilización de deportes adaptados y un encuentro paralímpico final, además de charlas y visionado de videos. Este programa toma la teoría del contacto y la teoría del comportamiento planificado (Ajzen, 1991) como base científica. La teoría del comportamiento planificado indica que las actitudes están influenciadas por nuestras creencias, basadas en nuestra experiencia, y la adquisición de información nueva. Al dotar a los participantes de experiencias positivas respecto a la discapacidad a través del deporte, se modifican sus creencias, que determinan a su vez las actitudes. Además, Pérez-Tejero, Ocete, Ortega-Vila y Coterón (2012), demostraron que los participantes en un campus de baloncesto inclusivo, en el que participaban personas con y sin discapacidad física, mejoraron las actitudes hacia la discapacidad.

Los programas de cambio de actitudes hacia la discapacidad en EF han utilizado diferentes tipos de para-deportes y tipos de discapacidad. Así, encontramos experiencias con deportes para personas con discapacidad física, como el baloncesto en silla de ruedas o la boccia (González y Baños, 2012; Pérez-Tejero y col., 2012) y para personas con discapacidad visual, como el goalball (Santana y Garoz, 2013) o el fútbol-5 paralímpico (Reina, López, Jiménez, García, y Hutzler, 2011). Sin embargo, las experiencias y contacto con personas con discapacidad intelectual (DI), como es el caso de esta investigación, practicando deporte inclusivo, entendido como práctica conjunta deportiva de personas con y sin discapacidad teniendo en cuenta las posibilidades de los participantes y conservando el objetivo de la modalidad deportiva (Pérez-Tejero, 2015), actividad física o EF junto a personas sin discapacidad son muy escasas (ver Abellán, Hernández-Martínez, González-Martí, y SerraOlivares, 2015 como excepción). 
Abellán, J.; Sáez-Gallego, N., y Reina, R. (2018). Explorando el efecto del contacto y el deporte inclusivo en Educación Física en las actitudes hacia la discapacidad intelectual de los estudiantes de secundaria. RICYDE. Revista internacional de ciencias del deporte. 53(14), 233-242. https://doi.org/10.5232/ricyde2018.05304

El objetivo del presente trabajo fue examinar el efecto de un programa de cambio de actitudes hacia la discapacidad a partir del contacto de estudiantes de secundaria con personas con discapacidad intelectual (DI), compartiendo práctica de deporte inclusivo. De forma más específica, se analizó el cambio las actitudes de estudiantes de Educación Secundaria Obligatoria (ESO) a partir de la práctica compartida de deporte inclusivo con personas con DI. Para obtener una imagen más completa del efecto en las actitudes de los participantes se crearon dos grupos experimentales distintos, el grupo Actividades (Grupo A) y el grupo Visita y Actividades (Grupo V-A), y un grupo control. Se partió de la hipótesis de que el grupo que realiza el contacto junto con la actividad de deporte inclusivo mejoraría sus actitudes en mayor magnitud que los otros dos grupos de estudio. La presente investigación presenta una perspectiva de trabajo novedosa, combinando dos cuestionarios de reciente publicación, con el fin de explorar su empleabilidad de manera conjunta en un contexto de EF en ESO.

\section{Método}

\section{Participantes}

Ochenta y tres $(\mathrm{N}=83)$ estudiantes de ESO de dos centros de Castilla - La Mancha $(15,18 \pm$ 0,89 años) formaron parte de este estudio, de los cuales 40 eran chicos $(15,23 \pm 0,15$ años) y 43 chicas $(15,13 \pm 0,13$ años). Atendiendo al curso en el que se encontraban en el momento de realización del estudio, encontramos que 32 estaban en $3^{\circ}\left(14,44 \pm 0,564\right.$ años), y 51 en $4^{\circ}$ de ESO (15,65 $\pm 0,74$ años). La selección de la muestra ha seguido un modelo no probabilístico de conveniencia, ya que los centros seleccionados para hacer el estudio fueron aquellos que tenían programadas las actividades (visita de instalaciones del centro ocupacional y/o actividad deportiva inclusiva) en colaboración con una asociación de personas con DI.

Los participantes fueron distribuidos en tres diferentes grupos de análisis, en función de las actividades en las que cada grupo se vio involucrado como parte del programa educativo desarrollado en su centro: un grupo Control ( $N=33,20$ chicas y 13 chicos; 15,45 $\pm 1,06$ años) y dos grupos experimentales: el grupo Actividad $(\mathrm{N}=25,12$ chicas y 13 chicos; 14,52 $\pm 0,58$ años) y el grupo Visita-Actividad ( $\mathrm{N}=25,11$ chicas y 14 chicos; 15,48 $\pm 0,51$ años). En el apartado de procedimiento se ofrece una información más detallada sobre las actividades desarrolladas por cada uno de los grupos.

Todos los estudiantes participaron en el estudio voluntariamente y aportaron la autorización firmada por su padre, madre o tutor legal antes de comenzar. Se obtuvieron además los permisos pertinentes del equipo de dirección de los dos centros donde se llevó a cabo la recolección de datos. La presente investigación respeta los acuerdos de la Declaración de Helsinki.

\section{Instrumentos}

Para el desarrollo del estudio se utilizaron dos instrumentos. El primero de ellos hace referencia a la versión en español del cuestionario "The Attitudes towards Inclusion of Students with Disabilities in Physical Education” (AISDPE) (Reina, Hutzler, Iñiguez-Santiago, y MorenoMurcia, 2016). Este cuestionario de actitudes hacia la inclusión de estudiantes con discapacidad en educación física se basa en una escala de 17 ítems que incluye dos componentes: el componente cognitivo (ítems 1, 3, 4, 6, 13, 14 y 15: e.g. Ítem 7: "Pienso que destacaría si participo con personas con discapacidad en una actividad fisica o deporte cualquiera") y el componente conductual (ítems 2, 5, 7, 8, 9, 10, 11, 12, 16 y 17: e.g. Ítem 17: "Prefiero no interaccionar con personas con discapacidad"). Los coeficientes $\alpha$ para cada una de las subescalas del AISDPE fueron de ,82 para el componente cognitivo y de ,75 para el componente conductual (Reina y col., 2016). 
Abellán, J.; Sáez-Gallego, N., y Reina, R. (2018). Explorando el efecto del contacto y el deporte inclusivo en Educación Física en las actitudes hacia la discapacidad intelectual de los estudiantes de secundaria. RICYDE. Revista internacional de ciencias del deporte. 53(14), 233-242. https://doi.org/10.5232/ricyde2018.05304

El segundo instrumento es la "Escala de Actitud hacia el Alumnado con Discapacidad en Educación Física” (EAADEF) (Iñiguez-Santiago, Férriz, Martínez-Galindo, CebriánSánchez, y Reina, 2017). Esta escala incluye cuatro ítems, todos ellos relacionados con el componente conductual (e.g. Ítem 1: "prefiero no relacionarme con personas con discapacidad"). La consistencia interna del instrumento, medida mediante el coeficiente $\alpha$, fue de ,77 (Íñiguez-Santiago y col., 2017).

Para la administración de ambos cuestionarios se empleó una escala Likert de 1 (totalmente en desacuerdo) a 5 (totalmente de acuerdo) y la sentencia previa "En las siguientes situaciones con personas con discapacidad...". Ambos cuestionarios están enunciados de manera que una mayor puntuación en el mismo significa una actitud menos favorable hacia la discapacidad y, por ende, menor predisposición a una situación educativa potencialmente inclusiva. En ambos cuestionarios se utilizó la media de las escalas como valor estadístico.

\section{Procedimiento}

Los equipos directivos de los centros educativos aprobaron la realización del estudio y posteriormente el profesorado implicado presentó su conformidad para participar en el mismo. Se administraron los cuestionarios a todos los estudiantes participantes, antes y después de realizar la intervención. Los participantes fueron divididos en tres grupos distintos de estudio: i) el grupo C (Control), que sólo completó cuestionarios en dos momentos temporales; ii) el grupo V-A, que realizó una visita a las instalaciones de un centro especial de empleo de personas con DI y realizó una jornada de deporte inclusivo junto al grupo de personas con DI que habían visitado; e iii) el grupo A, que realizó la misma actividad de deporte inclusivo que el grupo V-A. Los grupos V-A y A cumplimentaron los dos instrumentos de evaluación de las actitudes, tanto antes como después de sus respectivas intervenciones. El contacto con la muestra se perdió, por lo que no se pudo realizar un re-test para comprobar si los cambios se mantuvieron en el tiempo, lo que se considera una limitación del estudio.

La visita al centro se realizó durante un periodo de 2 horas, en el que los participantes, guiados por un profesional del centro, visitaron las instalaciones en las que trabajan más de 100 personas con DI. Durante este tiempo recibieron información sobre el tipo de trabajo que se realizaba y pudieron escuchar a algunos de los usuarios mientras describían su trabajo diario. La actividad deportiva inclusiva consistió en un encuentro en el que los dos grupos experimentales compartieron práctica con un grupo de deportistas con discapacidad intelectual (20 deportistas), en dicho encuentro se realizaron pruebas de atletismo (carreras de relevos) y un partido de fútbol sala. En ambos casos, los dos grupos de intervención realizaron una práctica conjunta con los deportistas con DI, en equipos inclusivos integrados por personas con y sin discapacidad.

La administración de los cuestionarios se realizó durante el tercer trimestre del curso académico en todos los grupos. El tiempo total de la intervención, entre la medida pre-test, los momentos de contacto (según el grupo) y el post-test, se realizó en un mes.

\section{Análisis de los datos}

Para la estadística inferencial se ha utilizado el ANOVA de medidas repetidas sobre las diferentes variables, tomando 0,05 como nivel de significación de Alfa. En todos los casos se ha introducido el grupo (Actividad, Visita-Actividad y Control) como factor inter-sujeto y el momento de evaluación (pre-test y post-test) como factor intra-sujeto. Se ha utilizado un modelo mixto de ANOVA de medidas repetidas, asumiendo que la varianza de las variables es igual (supuesto de esfericidad). Para evitar el error de tipo I, se ha utilizado el método de Huyn - Feldt como procedimiento de corrección, con el objetivo de ajustar los grados de libertad utilizados para la intervención y los efectos del error. Se ha calculado el tamaño del efecto 
Abellán, J.; Sáez-Gallego, N., y Reina, R. (2018). Explorando el efecto del contacto y el deporte inclusivo en Educación Física en las actitudes hacia la discapacidad intelectual de los estudiantes de secundaria. RICYDE. Revista internacional de ciencias del deporte. 53(14), 233-242. https://doi.org/10.5232/ricyde2018.05304

extrayendo el Eta cuadrado parcial $\left(\eta_{\mathrm{p}}{ }^{2}\right)$, siguiendo la siguiente interpretación: superior a 0,26 , entre 0,26 y 0,02 , y menor que 0,02 como grande, medio y pequeño respectivamente (Pierce, Block, y Aguinis, 2014).

Posteriormente, se ha utilizado como post-hoc la prueba $t$ de student para muestras relacionadas con las correcciones de Bonferroni, con el objetivo de observar qué grupos presentan diferencias tras la intervención en las variables en las que se obtiene una interacción significativa de los grupos a lo largo de los test. Adicionalmente, se aplicó el test de Bonferroni como post-hoc para explorar entre qué grupos existen diferencias en las variables en las que se observa un efecto significativo del grupo. Tanto para la estadística descriptiva como inferencial se ha utilizado SPSS 23 (SPSS Inc., Chicago, IL, USA).

\section{Resultados}

La Tabla 1 muestra los estadísticos descriptivos (media \pm desviación típica) de la puntuación obtenida en cada una de las dimensiones (AISDPE Cognitiva, AISDPE Conductual y EAADEF) por cada grupo, antes y después de la intervención, y la Figura 1 muestra el efecto de la intervención en las actitudes hacia la discapacidad en cada dimensión en función del grupo.

Tabla 1. Estadísticos descriptivos de los grupos para las actitudes hacia la discapacidad, antes y después de la intervención.

\begin{tabular}{|c|c|c|c|c|}
\hline \multirow[b]{2}{*}{ VARIABLES } & & \multicolumn{3}{|c|}{ GRUPOS } \\
\hline & & $\begin{array}{l}\text { Actividad } \\
(\mathrm{n}=25)\end{array}$ & $\begin{array}{c}\text { Visita-Actividad } \\
\qquad(\mathrm{n}=\mathbf{2 5})\end{array}$ & $\begin{array}{l}\text { Control } \\
(\mathbf{n}=\mathbf{3 3})\end{array}$ \\
\hline \multirow{2}{*}{$\begin{array}{l}\text { AISDPE } \\
\text { Cognitiva }^{\text {T I G }}\end{array}$} & Pre-test & $2,84 \pm 0,64$ & $2,74 \pm 0,50$ & $2,25 \pm 0,54$ \\
\hline & Post-test & $2,67 \pm 0,81$ & $2,43 \pm 0,49$ & $2,35 \pm 0,58$ \\
\hline \multirow{2}{*}{$\begin{array}{l}\text { AISDPE } \\
\text { Conductual }^{\mathrm{G}}\end{array}$} & Pre-test & $2,66 \pm 0,56$ & $2,44 \pm 0,57$ & $2,10 \pm 0,56$ \\
\hline & Post-test & $2,60 \pm 0,75$ & $2,32 \pm 0,62$ & $2,25 \pm 0,65$ \\
\hline \multirow[t]{2}{*}{ EAADEF $^{\mathrm{G}}$} & Pre-test & $2,38 \pm 0,89$ & $2,24 \pm 1,04$ & $1,77 \pm 0,80$ \\
\hline & Post-test & $2,39 \pm 0,99$ & $2,09 \pm 0,81$ & $1,89 \pm 0,79$ \\
\hline
\end{tabular}

Nota. Superíndice $\left({ }^{\mathrm{T}}\right)$ marca efecto significativo del test para $p<0,05$. Superíndice ${ }^{\mathrm{I}}$ ) marca interacción significativa Grupo $\mathrm{x}$ Test para $p<0,05$. Superíndice $\left({ }^{\mathrm{G}}\right)$ muestra diferencias significativas entre grupos para $p<0,05$. 
Abellán, J.; Sáez-Gallego, N., y Reina, R. (2018). Explorando el efecto del contacto y el deporte inclusivo en Educación Física en las actitudes hacia la discapacidad intelectual de los estudiantes de secundaria. RICYDE. Revista internacional de ciencias del deporte. 53(14), 233-242. https://doi.org/10.5232/ricyde2018.05304

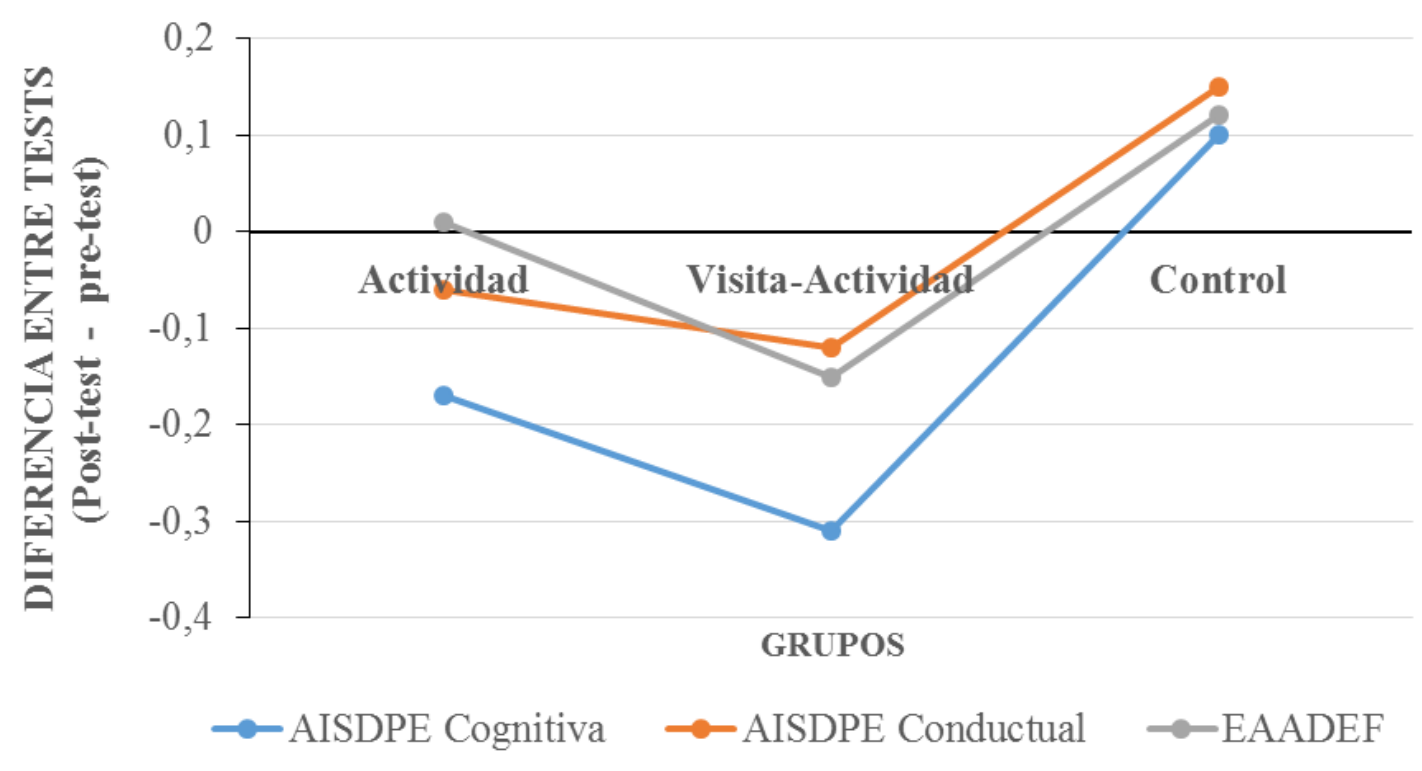

Figura 1. Efecto de la intervención en las actitudes hacia la discapacidad (Post-test - Pre-test).

El ANOVA de medidas repetidas muestra un efecto significativo del test en la dimensión AISDPE Cognitiva $\left[F(1,80)=5,715 ; p=0,019 ; \eta_{\mathrm{p}}^{2}=0,067\right]$. Además, existe una interacción significativa Grupo $\mathrm{x}$ Test en esta misma variable $\left[F(2,80)=5,723, p=0,005, \eta_{\mathrm{p}}{ }^{2}=0,125\right]$. La prueba $t$ de student para muestras relacionadas con las correcciones de Bonferroni, utilizada como post-hoc para cada uno de los grupos, desvela que es el grupo V-A el que propicia esa interacción significativamente diferente $[t(24)=4,561, p<0,001]$. Este grupo reduce significativamente su puntuación en el Post-test (disminuye su media en 0,31 puntos), lo que indica que mejora sus actitudes hacia la discapacidad en la dimensión AISDPE Cognitiva tras la intervención. Por su parte, el cambio producido por el grupo A (disminuye su media en 0,17 puntos) y el grupo $\mathrm{C}$ (aumenta su media en 0,10 puntos) no es significativo.

El ANOVA de medidas repetidas también muestra diferencias significativas entre algunos de los grupos al comparar las actitudes hacia la discapacidad. Estas diferencias se encuentran en las tres dimensiones: AISDPE Cognitiva $\left[F(2,80)=5,038, p=0,009, \eta_{\mathrm{p}}{ }^{2}=0,112\right]$; AISDPE Conductual $\left[F(2,80)=4,674, p=0,012, \eta_{\mathrm{p}}{ }^{2}=0,105\right]$; y EAADEF $[F(2,80)=3,572, p=0,033$, $\left.\eta_{\mathrm{p}}{ }^{2}=0,082\right]$. En todos los casos, las diferencias indican que el grupo Actividad difiere en sus actitudes significativamente respecto del grupo Control. Estas diferencias se pueden observar en la Tabla 2. 
Abellán, J.; Sáez-Gallego, N., y Reina, R. (2018). Explorando el efecto del contacto y el deporte inclusivo en Educación Física en las actitudes hacia la discapacidad intelectual de los estudiantes de secundaria. RICYDE. Revista internacional de ciencias del deporte. 53(14), 233-242. https://doi.org/10.5232/ricyde2018.05304

Tabla 2. Medidas marginales estimadas para las comparaciones entre grupos.

\begin{tabular}{cccccc}
\hline & Media & $\begin{array}{c}\text { Error } \\
\text { estándar }\end{array}$ & $\begin{array}{c}\text { Intervalo de confianza al 95\% } \\
\text { Límite } \\
\text { inferior }\end{array}$ & $\begin{array}{c}\text { Límite } \\
\text { superior }\end{array}$ \\
\hline \multirow{2}{\text{AISDPE}}{} & Actividad* & 2,757 & 0,110 & 2,538 & 2,976 \\
& Actividad-Visita & 2,583 & 0,110 & 2,364 & 2,802 \\
& Control* $^{\text {AISDPE }}$ & 2,304 & 0,096 & 2,113 & 2,494 \\
Conductual & Actividad-Visita & 2,380 & 0,113 & 2,156 & 2,604 \\
& Actividad* & 2,632 & 0,113 & 2,408 & 2,856 \\
& Control* & 2,175 & 0,098 & 1,980 & 2,370 \\
& & & & & 2,700 \\
EEADEF & Actividad* & 2,385 & 0,158 & 2,070 & 2,480 \\
& Actividad-Visita & 2,165 & 0,158 & 1,850 & 2,108 \\
\hline
\end{tabular}

Nota. Asterisco $(*)$ muestra diferencias significativas entre grupos para $p<0,05$.

\section{Discusión}

El objetivo del presente trabajo era explorar los cambios que la intervención, basada en el contacto con personas con DI a través de una visita a sus instalaciones y/o una actividad de deporte inclusivo junto a ellas, producen en las actitudes hacia la DI de los estudiantes de Educación Secundaria. A pesar de su notable presencia en la sociedad y de acuerdo con Scior (2011), existe un desconocimiento público de este tipo de discapacidad, posiblemente debido a que no han existido intervenciones bien diseñadas para reducir las actitudes negativas hacia la DI y a la falta de investigación al respecto.

Los resultados indican que el grupo V-A mostró un efecto positivo tras la realización del programa, en comparación con los grupos A y control. La práctica de deporte inclusivo y la visita al centro de trabajo consiguieron que los participantes del grupo V-A mejoraran significativamente sus actitudes hacia la discapacidad en la variable AISDPE cognitiva, aquella que representa la idea que los estudiantes pudieran tener hacia el colectivo de personas con DI. Estos resultados indican que aquellos estudiantes con un contacto más cercano con personas con DI pueden mejorar más sus actitudes hacia éstas. Por lo tanto, compartir experiencias deportivas inclusivas y conocer más acerca de la vida de las personas con DI se presenta como una forma de mejorar las actitudes hacia dicho colectivo. La práctica de deporte inclusivo durante las clases de EF ya había obtenido resultados positivos anteriormente (e.g. Santana y Garoz, 2013), pero no se había incluido hasta ahora el contacto con personas con DI.

Los estudios centrados en mejorar las actitudes hacia la DI no son muy abundantes. Beh-Pajooh (1991) observó la mejora de las actitudes hacia la discapacidad de estudiantes universitarios a través del contacto con personas con DI. Nosse y Gavin (1991) también mostraron un efecto positivo del contacto directo con personas con DI hacia las actitudes mostradas por estudiantes universitarios voluntarios. También Rillotta y Nettelbeck (2007) obtuvieron resultados positivos, si bien en este caso los estudiantes de secundaria mejoraron sus actitudes hacia la DI a partir de un programa de concienciación hacia dicha discapacidad. De igual manera que el grupo V-A en el presente trabajo, el trabajo de Schawartz y Rabinovitz (2001) incluyó una visita a las instalaciones de una residencia de personas con DI, y sus resultados indicaron que 
Abellán, J.; Sáez-Gallego, N., y Reina, R. (2018). Explorando el efecto del contacto y el deporte inclusivo en Educación Física en las actitudes hacia la discapacidad intelectual de los estudiantes de secundaria. RICYDE. Revista internacional de ciencias del deporte. 53(14), 233-242. https://doi.org/10.5232/ricyde2018.05304

la visita por sí sola no conseguía un efecto positivo en las actitudes, pero sí lo hacía combinada con otras variables (p.e. los participantes con hijos pequeños que visitaron las instalaciones sí mostraron mejoras).

En esta línea, aunque el grupo A mejora sus actitudes, estos cambios no llegan a ser significativos, lo que puede ser debido a las características de la intervención, concretamente al tiempo de contacto y a la información recibida durante éste. Al no realizar la visita, este grupo obtiene una menor información y un menor tiempo de contacto con las personas con DI si lo comparamos con el grupo V-A, lo que puede haber motivado el menor cambio de actitudes para el grupo A. Estos resultados están apoyados por los obtenidos en otras investigaciones, por lo que un mayor contacto con personas con discapacidad y mayor información al respecto propicia mejores actitudes hacia la discapacidad (Flórez, Aguado y Alcedo, 2009).

Por otra parte, cabe destacar las diferencias encontradas en las actitudes hacia la discapacidad en las tres escalas utilizadas entre los participantes que pertenecían al grupo Control y aquellos que pertenecían al grupo Actividad. En general, aunque el grupo control no ha modificado sus resultados como lo han hecho los otros dos grupos, éste mostraba una actitud hacia la discapacidad más favorable, siendo significativa al compararlo con las actitudes del grupo Actividad. Estas diferencias pueden estar motivadas por las características de los grupos, ya que el Control tiene un mayor porcentaje de chicas (60,6\%) que el grupo Actividad (48\%), y como se ha demostrado en investigaciones previas, las mujeres presentan de forma general una actitud más positiva hacia la discapacidad (García-Fernández, Inglés, Vicent, Gonzálvez, y Mañas, 2013; Litvack, Ritchie, y Shore, 2011).

Este estudio presenta una perspectiva novedosa, incluyendo el contacto con personas con DI en los programas de cambio de actitudes hacia la discapacidad en EF. Anteriormente, sólo el trabajo de Abellán y col. (2015) había seguido una forma de trabajo similar, pero en ese caso incluyendo el contacto con personas con DI para modificar las actitudes hacia la discapacidad de futuros maestros de EF, quienes debieron diseñar una sesión de EF para personas con DI en el punto final del programa de modificación de las actitudes. Sin embargo, el presente trabajo parte de la premisa de compartir experiencias a través del deporte inclusivo, mientras que en el trabajo de Abellán y col. (2015) estaba enfocado hacia la formación del profesorado de EF. A tenor de los resultados obtenidos se sugiere la incorporación de deporte inclusivo con personas con DI en los programas curriculares de EF en secundaria, de acuerdo con la idea de Robles-Rodríguez y col. (2017), quienes sugieren la incorporación de para-deportes o deportes adaptados como contenidos propios de EF en la Educación Secundaria.

\section{Conclusiones}

Teniendo en cuenta la naturaleza exploratoria de este trabajo, los objetivos y lo reducido de la muestra, podemos concluir, que en esta población de estudio la adquisición de nueva información sobre la DI, a partir de la visita a las instalaciones y la práctica de deporte inclusivo, ha sido una experiencia positiva, que ha conseguido modificar algunas de las actitudes hacia la DI de los estudiantes de Educación Secundaria incluidos en el grupo V-A. 
Abellán, J.; Sáez-Gallego, N., y Reina, R. (2018). Explorando el efecto del contacto y el deporte inclusivo en Educación Física en las actitudes hacia la discapacidad intelectual de los estudiantes de secundaria. RICYDE. Revista internacional de ciencias del deporte. 53(14), 233-242. https://doi.org/10.5232/ricyde2018.05304

\section{Referencias}

Abellán, J., y Hernández-Martínez, A. (2016). Los juegos motores sensibilizadores y su efecto en las actitudes hacia la discapacidad mostradas por futuros maestros de Educación Física Sportis Scientific Technical Journal, 2(1), 93-106.

Abellán, J.; Hernández-Martínez, A.; González-Martí, I., y Serra-Olivares, J. (2015). Efecto de un programa para mejorar las actitudes hacia la discapacidad en estudiantes del grado de Maestro de Primaria Mención de Educación Física. En M.T. Ramiro, T. RamiroSánchez y M.P. Bermúdez (Comp.), Evaluación de la Calidad de la Investigación y de la Educación Superior: Libro de Resúmenes XII FECIES (p. 476). Granada: Asociación Española de Psicología Conductual (AEPC).

Ajzen, I. (1991). The Theory of Planned Behavior. Organizational Behavior and Human Decision Processes, 50(2), 179-211. https://doi.org/10.1016/0749-5978(91)90020-T

Allport, G. (Ed.). (1954). The nature of prejudice. New York: Doubleday Books.

Beh-Pajooh, A. (1991). The effect of social contact on college students' attitudes toward severely handicapped students and their educational integration. Journal of Mental Deficiency Research, 35(4), 339-352.

https://doi.org/10.1111/j.1365-2788.1991.tb00406.x

Felipe, C., y Garoz, I. (2014). Actividad físico-deportiva en programas de cambio de actitudes hacia la discapacidad en edad escolar: Una revisión de la literatura. Cultura, Ciencia y Deporte, 27(9), 199-210.

Flórez, M. A.; Aguado, A. L., y Alcedo, M. A. (2009). Revisión y análisis de los programas de cambio de actitudes hacia personas con discapacidad. Anuario de Psicología Clínica y de la Salud, 5, 85-98.

García-Fernández, J. M.; Inglés, C. J.; Vicent, M.; Gonzálvez, C., y Mañas, C. (2013). Actitudes hacia la Discapacidad en el Ámbito Educativo a través del SSCI (2000-2011). Análisis Temático y Bibliométrico. Electronic Journal of Research in Educational Psychology, 11(1), 139-166.

González, J., y Baños, L. M. (2012). Estudio sobre el cambio de actitudes hacia la discapacidad en clases de actividad física. Cuadernos de Psicología del Deporte, 12(2), 101-108. https://doi.org/10.4321/S1578-84232012000200011

Íñiguez-Santiago, M. C.; Férriz, R.; Martínez-Galindo, M. C.; Cebrián-Sánchez, M., y Reina, R. (2017). Análisis factorial de la escala de actitudes hacia el alumnado con discapacidad en educación física (EAADEF). Psychology, Society, \& Education, 9 (3), 493-504.

IPC. (2004). Paralympic School Day Manual. Recuperado el 08/02/2017 de https://www.paralympic.org/the-ipc/paralympic-school-day

Litvack, M. S.; Ritchie, K. C., \& Shore, B. M. (2011). High and average-achieving students' perceptions of disabilities and of students with disabilities in inclusive classrooms. Exceptional Children, 77(4), 474-487.

McKay, C.; Block, M., \& Parck, J.Y. (2015). The impact of paralympic school day on student attitudes toward inclusion in physical education. Adapted Physical Activity Quarterly, 32(4), 331-348.

https://doi.org/10.1123/APAQ.2015-0045

Nosse, L. J., \& Gavin, K. J. (1991). Influence of direct contact on college students' attitude toward adults with mental handicaps. College Student Journal, 25(2), 201-206.

Ocete, C.; Pérez-Tejero, J., y Coterón, J. (2015). Propuesta de un programa de intervención educativa para facilitar la inclusión de alumnos con discapacidad en 
Abellán, J.; Sáez-Gallego, N., y Reina, R. (2018). Explorando el efecto del contacto y el deporte inclusivo en Educación Física en las actitudes hacia la discapacidad intelectual de los estudiantes de secundaria. RICYDE. Revista internacional de ciencias del deporte. 53(14), 233-242. https://doi.org/10.5232/ricyde2018.05304

educación física. Retos. Nuevas tendencias en Educación Física, Deporte y Recreación, $27,140-145$.

Pérez-Tejero, J. (2015). El centro de estudios sobre deporte inclusivo (CEDI): 6 años de recorrido en investigación y formación para la inclusión social. En J. Gallego, M. AlcarazIbáñez, J. M. Aguilar-Parra y A. J. Cangas (Eds). IV Congreso Internacional de Deporte Inclusivo. Libro de Actas (pp. 14 -33). Almería: Universidad de Almería.

Pérez-Tejero, J., Ocete, C., Ortega-Vila, G., y Coterón, J. (2012). Diseño y aplicación de un programa de intervención de práctica deportiva inclusiva y su efecto sobre la actitud hacia la discapacidad: El Campus Inclusivo de Baloncesto. RICYDE. Revista Internacional de Ciencias del Deporte, 29(8), 258-271. https://doi.org/10.5232/ricyde2012.02905

Pierce, C. A; Block, R. A, \& Aguinis, H. (2004). Cautionary note on reporting eta-squared values from multifactor ANOVA designs. Educational and Psychological Measurement, 64(6), 916-924. https://doi.org/10.1177/0013164404264848

Reina, R.; Hutzler, Y.; Iñiguez-Santiago, M. C., \& Moreno-Murcia, J. A. (2016). Attitudes towards inclusion of students with disabilities in Physical Education Questionnaire (AISDPE): A two-component scale in Spanish. European Journal of Human Movement, 36, 75-87.

Reina, R.; López, V.; Jiménez, M.; García, T., \& Hutzler, Y. (2011). Effects of awareness interventions on children's attitudes toward peers with a visual impairment. International Journal of Rehabilitation Research, 34(3), 235-242. https://doi.org/10.1097/MRR.0b013e3283487f49

Rillotta, F., \& Nettelbeck, T. (2007). Effects of an awareness program on attitudes of students without an intellectual disability towards persons with an intellectual disability. Journal of Intellectual and Developmental Disability, 32(1), 19-27. https://doi.org/10.1080/13668250701194042

Robles-Rodríguez, J.; Abad-Robles, M.T.; Giménez, J., y Benito-Peinado, P. (2017). Los deportes adaptados como contribución a la educación en valores y a la mejora de las habilidades motrices: la opinión de los alumnos de Bachillerato. Retos, 31, 140-144.

Santana, P., y Garoz, I. (2013). Actitudes hacia la discapacidad e intervención docente desde el deporte adaptado. Revista Internacional de Medicina y Ciencias de la Actividad Física y el Deporte, 49(13), 1-17.

Schwartz, C., \& Rabinovitz, S. (2001). Residential facilities in the community for people with intellectual disabilities: How neighbours' perceptions are affected by the interaction of facility and neighbour variables. Journal of Applied Research in Intellectual Disabilities, 14(2), 100-109. https://doi.org/10.1046/j.1468-3148.2001.00060.x

Scior, K. (2011). Public awareness, attitudes and beliefs regarding intellectual disability: A systematic review. Research in Developmental Disabilities, 32(6), 2164-2182. https://doi.org/10.1016/j.ridd.2011.07.005 